\title{
GEOMETRICAL REALIZATION OF COMBINATORIAL GEOMETRIES
}

\author{
J. H. MASON
}

ABstract. A method of depicting combinatorial geometries geometrically is used to construct examples of small combinatorial geometries which arise as subsets of a vector space over division rings restricted by their characteristic.

A combinatorial pregeometry $(E, \mathcal{C})$ consists of a finite set $E$ and an identified collection $\mathfrak{C}$ of subsets called circuits satisfying the property that if $C_{1}$ and $C_{2}$ are distinct members of $\mathrm{e}$, then $C_{1}$ is not contained in $C_{2}$, and if $e$ is a member of $C_{1} \cap C_{2}$, then there exists a circuit $C$ contained in $C_{1} \cup C_{2}-\{e\}$. A subset of $E$ is called independent if it contains no circuit. It is well known (Whitney [7], Crapo-Rota [1]) that all maximal independent subsets of $E$ have the same cardinality, called the rank of $E$, and that an integer valued rank function is induced on the subsets of $E$ satisfying:

(i) $A \subseteq B$ implies rank $A \leqq$ rank $B$.

(ii) rank $A+\operatorname{rank} B \geqq \operatorname{rank} A \cup B+\operatorname{rank} A \cap B$.

(iii) rank $\varnothing=0$.

(iv) rank $A \cup\{e\} \leqq \operatorname{rank} A+1$.

A combinatorial geometry (more briefly geometry) is a combinatorial pregeometry in which all circuits have cardinality at least three. As an example the subsets of a fixed subset $E$ of a vector space, which are minimal with respect to being linearly dependent, form the circuits of a pregeometry on $E$. A geometry which arises in this way is called representable, and a fundamental problem is to classify these geometries which are representable over some division ring.

The observation has been made many times (Mac Lane [5], Ingleton [3]) that a projective plane induces a geometry on its points by taking as circuits all sets of three collinear points, and all sets of four coplanar points, no three of which are collinear. It led Ingleton to conjecture that the smallest geometry (in cardinality) which cannot be represented (over any division ring) must have ten points, namely a Desarguesian configuration with one line missing.

It is frequently useful to depict portions of projective planes by

Received by the editors July 30, 1969 .

AMS 1969 subject classifications. Primary 0535, 0527.

Key words and phrases. Combinatorial geometry, representation, vector space, division ring, rank, circuit, Euclidean space, nonrepresentable geometry.

Copyright (c) 1971, American Mathematical Society 
drawing points in the plane and drawing curves through the sets of points which are collinear. One purpose of this note is to generalize this idea by showing that any combinatorial geometry can be depicted as a finite set of points lying on certain surfaces in Euclidean space. The Euclidean dimension of the surfaces helps to distinguish the rank of the set of points lying on that surface.

The second purpose of this note is to exhibit a number of interesting geometries, among them a class of Lazarson rank three geometries (Example 3) which are geometries whose representations require division rings with restricted characteristic [4], and a counterexample to Ingleton's conjecture (Example 1). Other counterexamples have been obtained by Vamös [6] and Crapo [2].

Theorem 1 provides a messy looking condition on a collection of families of subsets of a set $E$, which induces a geometry on the set $E$. The motivation for the condition is that it enables us to depict the combinatorial geometry geometrically. This is brought out in the useful special cases of rank 3 and rank 4 geometries.

Theorem 2 is the converse of Theorem 1, showing that every geometry is induced by a family of sets satisfying Theorem 1 .

THEOREM 1. Let $E$ be a finite set and for each $t, 3 \leqq t \leqq n+2$, let $P(t)$ denote a family of subsets of $E$ satisfying the following condition.

(*) If $A_{i} \in P\left(t_{i}\right)$ and $A_{1} \cap A_{2} \neq \varnothing$ then $A_{1} \cup A_{2} \subseteq A \in P(\lambda)$ where $\lambda$ is given by

$$
\begin{aligned}
\lambda & =t_{1}+t_{2}-\left|A_{1} \cap A_{2}\right|-1 \text { if }\left\{r:\left|A_{1} \cap A_{2}\right| \geqq r, A_{1} \cap A_{2} \subseteq A \in P(r)\right\}=\varnothing, \\
& =t_{1}+t_{2}-\min \left\{r:\left|A_{1} \cap A_{2}\right| \geqq r, A_{1} \cap A_{2} \subseteq A \in P(r)\right\} \quad \text { otherwise }
\end{aligned}
$$

and $P(n+2)=E$.

Then $(E, \mathcal{C})$ is a geometry with rank $E \leqq n+1$ where $\mathfrak{C}$ consists of the minimal members (under inclusion) of $\{C: C \subseteq A \in P(t)$ and $|C| \geqq t\}$.

The members of $P(t)$ turn out to be dependent subsets of $(E, \mathfrak{e})$ maximal with respect to having rank $t-1$, and the formula for $\lambda$, which states how these sets fit together is just a restatement of the submodular property of the rank function.

Proof. Let $C_{i} \subseteq A_{i} \in P\left(t_{i}\right)$ be members of $\mathcal{e}$. Then $\left|C_{i}\right|=t_{i}$ and if $e \in C_{1} \cap C_{2}, C_{1} \neq C_{2}$, then to verify the circuit axioms, we need only show that $D=C_{1} \cup C_{2}-\{e\}$ contains a member of $\mathcal{C}$. Now we are given that $D \subseteq A \in S(\lambda)$ where $\lambda$ is given by the formula. If $\lambda=t_{1}+t_{2}$ $-r$, then $\left|C_{1} \cap C_{2}\right| \leqq r-1$ since $C_{1} \cap C_{2}$ does not contain a member of e by minimality. Then since $|D|=t_{1}+t_{2}-1-\left|C_{1} \cap C_{2}\right|$ we get $|D| \geqq t_{1}+t_{2}-r=\lambda$. And if $\lambda=t_{1}+t_{2}-\left|A_{1} \cap A_{2}\right|-1$ then $\left|C_{1} \cap C_{2}\right|$ $\leqq\left|A_{1} \cap A_{2}\right|$ whence $|D| \geqq \lambda$. Thus in either case $D$ contains a member 
of e. Since $P(n+2)=E$, every subset of $E$ with cardinality $n+2$ is dependent, and so a basis of $(E, \mathcal{C})$ must have cardinality $\leqq n+1$.

THEOREM 2. Let $(E, \mathcal{C})$ be a combinatorial geometry and denote by $P(t)$ the family of maximal members of $\{A \subseteq E$ :rk $A=t-1<|A|\}$. Then the collection of families $P(t), 3 \leqq t \leqq \mathrm{rk} E+1$, satisfy the condition $(*)$.

Proof. Since $(E, \mathcal{C})$ is a geometry, $P(1)=P(2)=\varnothing$. We are given that rank $A_{i}=t_{i}-1,\left|A_{i}\right| \geqq t_{i}$ and $A_{1} \cap A_{2} \neq \varnothing$. Put $W\left(A_{1} \cap A_{2}\right)$ $=\left\{r:\left|A_{1} \cap A_{2}\right| \geqq r, A_{1} \cap A_{2} \subseteq A \in P(r)\right\}$. We need to verify that

(i) if $W\left(A_{1} \cap A_{2}\right)=\varnothing$ then rank $A_{1} \cup A_{2}=t_{1}+t_{2}-\left|A_{1} \cap A_{2}\right|-2$ $<\left|A_{1} \cup A_{2}\right|$ and

(ii) if $W\left(A_{1} \cap A_{2}\right) \neq \varnothing$ then rank $A_{1} \cup A_{2}=t_{1}+t_{2}-\min W\left(A_{1} \cap A_{2}\right)$ $-1<\left|A_{1} \cup A_{2}\right|$.

To establish (ii), let $t=\min W\left(A_{1} \cap A_{2}\right)$ and note that rank $A_{1} \cap A_{2}$ $=t-1$. Since $\left|A_{i}\right| \geqq\left|A_{1} \cap A_{2}\right|+t_{i}-t$ we find that

$$
\left|A_{1} \cup A_{2}\right| \geqq t_{1}+t_{2}-2 t+\left|A_{1} \cap A_{2}\right| \geqq t_{1}+t_{2}-t \text {. }
$$

Now let $X$ be a maximal independent subset of $A_{1} \cup A_{2}$ containing a maximal independent subset of $A_{1} \cap A_{2}$. The maximality of the $A_{i}$ in the definition of $P\left(t_{i}\right)$ assures us that

$$
|X| \geqq t_{1}-1+t_{2}-1-(t-1)=t_{1}+t_{2}-t-1
$$

Suppose by way of contradiction, that $|X| \geqq t_{1}+t_{2}-t$. Then we would have

$$
\begin{aligned}
\left|X \cap A_{1} \cap A_{2}\right| & =\left|X \cap A_{1}\right|+\left|X \cap A_{2}\right|-\left|X \cap\left(A_{1} \cup A_{2}\right)\right| \\
& \leqq t_{1}-1+t_{2}-1-\left(t_{1}+t_{2}-t\right) \leqq t-2 .
\end{aligned}
$$

Thus rank $A_{1} \cap A_{2} \leqq t-2 \leqq\left|A_{1} \cap A_{2}\right|$ contradicting the minimality of $t$.

Therefore rank $A_{1} \cup A_{2}=|X|=t_{1}+t_{2}-t-1$ and (ii) is established.

To verify (i), a similar argument shows that

$$
\text { rk } A_{1} \cup A_{2} \geqq t_{1}+t_{2}-\left|A_{1} \cap A_{2}\right|-2
$$

and

$\operatorname{rank} A_{1} \cup A_{2} \leqq \operatorname{rank} A_{1}+\operatorname{rank} A_{2}-\operatorname{rank} A_{1} \cap A_{2}$

$$
\leqq t_{1}-1+t_{2}-1-\left|A_{1} \cap A_{2}\right|<\left|A_{1} \cup A_{2}\right| \text {. }
$$

To check that the collection $P(t)$ does induce the original geometry $(E, \mathcal{C})$ we notice that $X$ contains a circuit induced from $P(t)$ if and only if $|X|>\operatorname{rk} X$, i.e. if and only if $X$ contains a member of $\mathfrak{e}$. 
Geometric realization. Suppose that for $3 \leqq t \leqq n+2, S(t)$ is a family of surfaces with Euclidean dimension $t-2$. Further, let $E$ be a finite set of points and suppose the collection $P(t)=E \cap S(t)$, $3 \leqq t \leqq n+2$, satisfy condition $\left(^{*}\right)$. Then by virtue of Theorem 1 , we can say that the collection of surfaces $S(t), 3 \leqq t \leqq n+2$, induce a geometry on $E$. The content of Theorem 2 is that every geometry can be so realized since the only restrictions on the surfaces $S(t)$ are their pairwise intersections in the finite set $E$. The following two special cases illustrate the situation for rank 3 and rank 4 geometries.

Special case: rank 3. Let $E$ be a finite set of points in the Euclidean plane, $S(3)$ a set of curves in the plane such that any two distinct curves meet in at most one point of $E$, and $S(4)$ the plane. Then the induced geometry $(E, \mathfrak{e})$ has rank $\leqq 3$.

Proof. Calculation based on the given intersections shows that, for $3 \leqq t_{1} \leqq t_{2} \leqq 4, \lambda=4$, i.e. that the union of any two distinct intersecting "surfaces" must lie in $S(4)$, which they do by definition.

Special case: rank 4. Let $E$ be a finite set of points in Euclidean 3space. Let $S(5)$ be the whole 3 -space, $S(4)$ a set of surfaces and $S(3)$ a set of curves satisfying:

(i) any two distinct surfaces which meet in more than two points of $E$ do so in a curve in $S(3)$;

(ii) any distinct curves which intersect in a point of $E$ do so in at most one and lie on a common surface;

(iii) any curve not lying on a surface intersects it in at most one point of $E$.

Then the induced space has rank $\leqq 4$.

PROOF. Using the intersection information, one calculates the table of values and checks that the two surfaces represented by $t_{1}$ and $t_{2}$ do lie on the surface represented by $\lambda$.

\begin{tabular}{|l|l|l|}
\hline \multicolumn{1}{|c|}{$t_{1}$} & $t_{2}$ & \multicolumn{1}{|c|}{$\lambda$} \\
\hline 3 & 3 & 4 \\
3 & 4 & 4,5 \\
4 & 4 & 5 \\
\hline
\end{tabular}

EXAmple 1. (Counterexample to Ingleton's conjecture.) Let $E=\left\{A, B, C, D, A^{\prime}, B^{\prime}, C^{\prime}, D^{\prime}\right\}$ where $A, B, C, D$ are the vertices of a regular tetrahedron in 3-space, and $A^{\prime}, B^{\prime}, C^{\prime}, D^{\prime}$ are the centroids of the opposite faces, respectively. Put $S(5)=3$-space, $S(4)=$ planes of 3-space except the plane through $A B A^{\prime} B^{\prime}$, and $S(3)=\varnothing$. The 
induced rank 4 cardinality 8 geometry $(E, \mathfrak{e})$ cannot be represented over any division ring.

Proof. Theorem 1 guarantees that we have a geometry. Assume that it is representable over a division ring $R$ in a left vector space. Without loss of generality we may select a basis of the vector space so that $A=(1,0,0,0), B=(0,1,0,0), C=(0,0,1,0), D=(0,0,0,1)$ and $C^{\prime}=(1,1,0,1)$. Since $\left\{A, B, C, D^{\prime}\right\}$ and $\left\{D, C, D^{\prime}, C^{\prime}\right\}$ are circuits, we can choose $D^{\prime}=(1,1, x, 0)$ where $0 \neq x \in R$. Then the circuits $\left\{A^{\prime}, B, C, D\right\},\left\{A, C, A^{\prime}, C^{\prime}\right\}$ and $\left\{A, D, A^{\prime}, D^{\prime}\right\}$ force $A^{\prime}=(0,1, x, 1)$ and similarly $B^{\prime}=(1,0, x, 1)$. But then $\left\{A, B, A^{\prime}, B^{\prime}\right\}$ would be linearly dependent contradicting our construction. Choosing $S(4)^{\prime}=S(4) \cup\{$ inscribed sphere $\}$, a distinct rank 4 geometry is induced which also cannot be represented.

REMARK. The fact that any nonrepresentable geometry must have at least eight points can be verified using the enumeration of all eight point and smaller geometries [2], or by a more tedious enumeration based on Theorem 2 .

Example 2. Let $E=\left\{A, B, C, A^{\prime}, B^{\prime}, C^{\prime}, X, Y, Z\right\}$ be nine points in the plane as shown in Figure 1. Put $S(4)=$ plane.

$S(3)=$ solid lines, inducing $(E, \mathfrak{e})$,

$S(3)^{*}=S(3)$ with a circle through $C, C^{\prime}, X$ inducing $\left(E, \mathfrak{e}^{*}\right)$,

$S(3)^{* *}=S(3)^{*}$ with a circle through $A, A^{\prime}, Z$, inducing $\left(E, \mathfrak{e}^{* *}\right)$,

$S(3)^{* * *}=S(3)^{*}$ with the line through $X, Y, Z$, inducing $\left(E, \mathrm{e}^{* * *}\right)$.

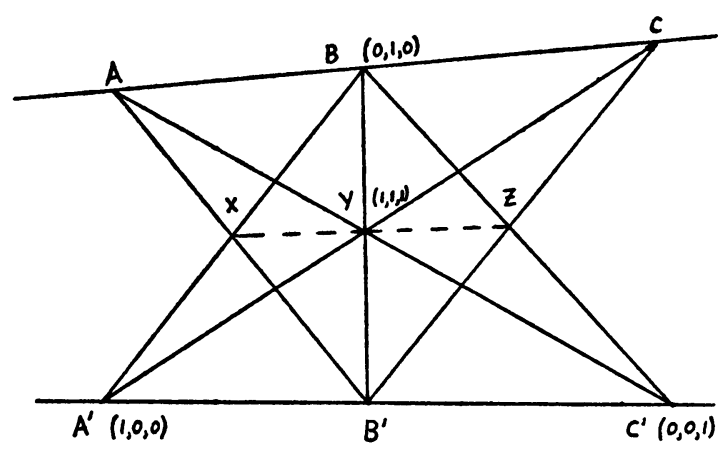

FIGURE 1

Then none of the four distinct rank 3 cardinality 9 geometries can be represented over any division ring.

ProOF. Assume they were representable. Without loss of generality $A^{\prime}, B, C^{\prime}$ and $Y$ can be chosen as shown. Let $X=(1, x, 0)$ for some $x$ in the division ring. Then one gets $A=(x, x, x-1), C=(x, x-1, x-1)$ 
and $Z=(0, x-1,-1)$ whence $X, Y, Z$ would be linearly dependent contradicting the construction of the first three geometries. Also, $\left\{C, C^{\prime}, X\right\}$ is a circuit if and only if $x^{2}-x+1=0$, and $A, A^{\prime}, Z$ is a circuit if and only if $x^{2}-x+1=0$. Thus $\left(E, \mathrm{e}^{* * *}\right)$ is not representable either.

EXAMPLE 3. (Lazarson geometries.) Let $E_{n}=\{M, P, Q, R, X, Y, Z\}$ $\cup\left\{A_{i}, B_{i}: 1 \leqq i \leqq n\right\}$ be $7+2 n$ points in the plane as shown in Figure 2 , with $S(4)=$ plane, $S(3)=$ lines shown. Without loss of generality, in any representation we may choose $X, Y, Z, M$ and hence $P, Q, R$ as shown. One easily deduces that $A_{i}=(1, i+1, i)$ and $B_{i}=(1, i, i+1)$. By adjoining to $S(3)$ a curve through $A_{i}, B_{i}, Z$ we induce a geometry on $E_{n}$ which requires the characteristic of the division ring to divide

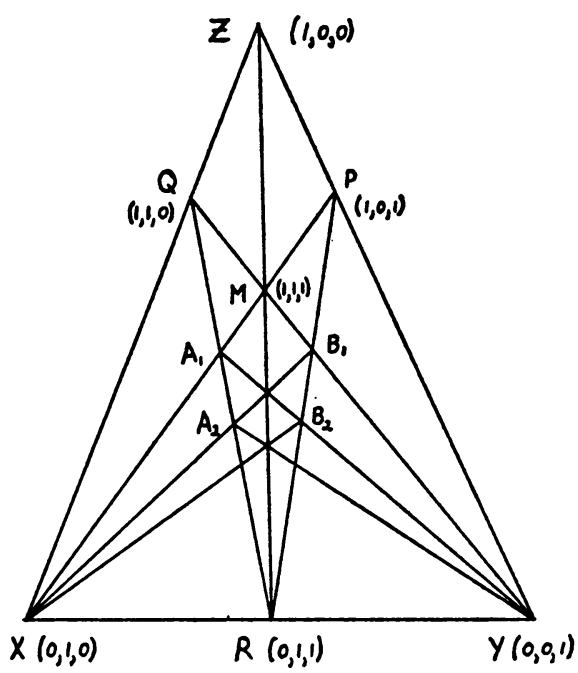

FIGURE 2

$2 i+1$. By taking two such curves, corresponding to $i$ and $j$ with $2 i+1,2 j+1$ distinct primes, the induced geometry becomes nonrepresentable. Thus we have geometries with cardinality $6+p$, rank 3 requiring characteristic $p$ (for odd primes $p$ )-improving Lazarson's examples with cardinality $2 p+3$, rank $p+1$ requiring characteristic $p$.

An outstanding problem is to find a geometry which is representable, but only over two distinct characteristics. Example 3 does not succeed because if you choose $2 i+1$ to be divisible by two primes $p$ and $q$, then the geometry is not representable over the larger prime. 
(If it were, we would have the point $A_{p}=(1, p+1, p)=(1,1,0)=Q$ which is not the case.)

The author would like to thank the referee for his many useful comments and suggestions.

\section{BIBLIOGRAPHY}

1. H. H. Crapo and G.-C. Rota, Combinatorial geometries, privately printed, December 1968.

2. J. E. Blackburn, H. H. Crapo and D. A. Higgs, A catalogue of combinatorial geometries (unpublished).

3. A. W. Ingleton, A note on independence functions and rank, J. London Math. Soc. 34 (1959), 49-56. MR 21 \#655.

4. T. Lazarson, The representation problem for independence functions, J. London Math. Soc. 33 (1958), 21-25. MR 20 \#5156.

5. S. Mac Lane, Some intepretations of abstract linear dependence in terms of projective geometry, Amer. J. Math. 28 (1937), 22-32.

6. P. Vamös, Private communication via R. A. Brualdi, July 1969.

7. $\mathrm{H}$. Whitney, On the abstract properties of linear dependence, Amer. J. Math. 57 (1935), 509-533.

University of Wisconsin, Madison, Wisconsin 53706

Open University, Bletchley, Bucks, United Kingdom 